\title{
Using Speckle-Tracking Echocardiography to Evaluate Subclinical Left Ventricular Dysfunction in Patients with Obstructive Sleep Apnea Syndrome
}

\author{
İbrahim Ersoy ${ }^{1}$ \\ 'Department of Cardiology, Faculty of Medicine, Afyonkarahisar Health Sciences University, Afyonkarahisar, Turkey \\ ORCID: \\ Ibrahim Ersoy: https://orcid.org/0000-0002-9553-8801
}

\section{Abstract}

Purpose: This study aims to evaluate left ventricular (LV) functions using speckle-tracking echocardiography (STE)-based analyses in the early stages of obstructive sleep apnea (OSA). Methods: Twenty-one healthy individuals and 79 OSA patients enrolled in the study. The OSA group was classified according to Apnea-Hypopnea Index (AHI) as mild, moderate, and severe. Two-dimensional tissue Doppler imaging and STE was performed. Results: The control and OSA groups were comparable for sex $(P=0.450)$ and age $(P=0.560)$, while diabetes $(P<0.001)$ and hypertension $(P<0.001)$ diagnoses and body mass index $(P<0.001)$ were higher in the OSA patients. In OSA group, global longitudinal strain (GLS) $(-13.32 \pm 3.19 \%, P<0.001)$, global circumferential strain (GCS) $(-18.33 \pm 3.40 \%, P<0.001)$ and global radial strain (GRS) $(37.91 \pm 8.11 \%, P=0.005)$ were reduced. GLS and GCS have a decreasing trend toward severe OSA, while GRS increased in mild and moderate OSA. According to linear regression analysis, GRS $(\beta:-1.47, P=0.001]$, GCS $(\beta:-1.34, P=0.001)$, GLS $(\beta:-1.54, P<0.001)$, systolic pulmonary artery pressure $(\beta: 0.99, P=0.001)$, and deceleration time $(\beta: 0.13, P=0.03)$ have an independent relationship with AHI. Conclusions: In our study, STE technique can be a practical method for manifesting LV functional impairment at the early stages of OSA.

Keywords: Global strain parameters, left ventricular dysfunction, obstructive sleep apnea, speckle-tracking echocardiography

\section{INTRODUCTION}

Obstructive sleep apnea (OSA) is a syndrome characterized by recurrent apnea and hypopnea episodes during sleep, primarily affecting the middle-aged population. ${ }^{[1]}$ Global estimates suggest that 936 million people worldwide have mild-to-moderate OSA, and 425 million have moderate-to-severe OSA. ${ }^{[2]}$ In some observational studies, the prevalence of OSA may be as high as $50 \%$ among patients with heart failure. OSA can play a crucial role in heart failure by causing repetitive hypoxia and apnea episodes, hormonal and metabolic impairment, systemic inflammation, and mechanical hemodynamic disturbances.

Extensive research has shown the link between OSA and cardiac remodeling causing heart failure. For example,

Received: 28-12-2020 Revised: 08-02-2021 Accepted: 19-02-2021 Published Online: 29-03-2021

\begin{tabular}{|l|l|}
\hline \multicolumn{3}{|c|}{ Access this article online } \\
\hline Quick Response Code: & Website: \\
& \\
http://www.ijcva.com
\end{tabular}

Borrelli et al. found a $62 \%$ prevalence of OSA in heart failure. ${ }^{[3]}$ Though, these results were based on basic echocardiographic parameters. Most of the studies have focused on left ventricular (LV) hypertrophy, decreased LV ejection fraction (EF), and LV diastolic dysfunction in the presence of OSA and heart failure. ${ }^{[3-5]}$

The strain is a measure of myocardial deformation. Furthermore, strain analysis is defined as a current LV function index method in recent echocardiography guidelines. ${ }^{[6,7]}$ There

Address for correspondence: Dr. ibrahim Ersoy, Department of Cardiology, Faculty of Medicine, Afyonkarahisar Science of Health University, Zafer Saglik Kulliyesi, Dortyol Mahallesi, 2078, No: 03, Afyonkarahisar, Turkey. E-mail: iersoytr@hotmail.com

This is an open access journal, and articles are distributed under the terms of the Creative Commons Attribution-NonCommercial-ShareAlike 4.0 License, which allows others to remix, tweak, and build upon the work non-commercially, as long as appropriate credit is given and the new creations are licensed under the identical terms.

For reprints contact: WKHLRPMedknow_reprints@wolterskluwer.com

How to cite this article: Ersoy I. Using speckle-tracking echocardiography to evaluate subclinical left ventricular dysfunction in patients with obstructive sleep apnea syndrome. Int J Cardiovasc Acad 2021;7:26-32. 
are three different types of strain reflecting cardiac axes: global longitudinal strain (GLS), circumferential, longitudinal strain (global circumferential strain [GCS]), and global radial strain (GRS). ${ }^{[8]}$ LV GLS has been introduced as a relevant clinical marker of LV function. ${ }^{[9]}$ To date, some studies have investigated the LV mechanics in patients with OSA using speckle-tracking echocardiography (STE) ${ }^{[10]}$

This paper's central hypothesis is that OSA with normal LV EF would have impairment in myocardial deformation. This situation could result in diastolic dysfunction in the early stages of the disease. Based on this hypothesis, our study has three main objectives: (1) to evaluate subclinical LV dysfunction with STE; (2) to investigate LV diastolic parameters and hypertrophic changes in OSA patients compared to a healthy control group; and (3) to ascertain determinant factors for Apnea-Hypopnea Index (AHI).

\section{Methods}

\section{Patients}

For this study, 88 consecutive newly diagnosed OSA patients aged between 18 and 100 in the Respiratory Medicine Division of Suleyman Demirel University Hospital were evaluated between January 2013 and January 2014. Patients with any of the following conditions were excluded: concomitant lung disease (including chronic obstructive pulmonary disease, bronchial asthma, or intestinal lung disease); heart failure (typical symptoms and signs, depressed LV EF, and elevated natriuretic peptides); valvular heart disease (hemodynamically significant regurgitation/stenosis); coronary heart disease (history of percutaneous intervention or coronary artery bypass graft surgery); atrial fibrillation or any arrhythmia; chronic renal and hepatic disease; a history of malignancy; or a suboptimal echo window. Nine individuals were excluded from the study based on concomitant lung disease $(n=5)$, a poor echocardiographic image $(n=3)$, and atrial fibrillation $(n=1)$, leaving 79 OSA patients (48 males and 31 females).

The control group was composed of 21 volunteers who were first admitted to the respiratory sleep clinic with an AHI of less than five and found normal cardiovascular examination by an experienced cardiologist between December 2014 and January 2014. Moreover, they did not meet the exclusion criteria.

The study was conducted under the Declaration of Helsinki. The Ethical Committee approved the study for Clinical Studies of Suleyman Demirel University (Registration Number: 181). All participants gave written informed consent.

\section{Polysomnography}

Polysomnography (PSG) was performed in a quiet, partially soundproof room with stable humidity and temperature. Data were examined with a Compumedics (44-channel E-series, Australia) PSG device. Electroencephalography, two-channel electrooculography, electromyography, oronasal airflow, thoracic and abdominal movements, body position, and pulse oximetry (oxygen saturation measured from the fingertip) were monitored during the procedure. According to the American Sleep Disorders Association criteria, an experienced chest disease specialist scored the PSG results. ${ }^{[1]}$

Apnea is defined as a cessation of the airflow signal for $10 \mathrm{~s}$ per hour. Hypopnea is identified as a $\geq 30 \%$ decrease in the airflow signal, associated with cortical arousal or oxygen desaturation of $\geq 3 \%$. The number of apnea plus hypopnea incidents per hour of sleep is calculated as the AHI. Patients with an AHI of less than five were considered OSA negative. This index was used to stratify OSA severity, as mentioned above.

\section{Standard echocardiographic measurements}

In the standard echocardiographic examination, patients were placed in the left lateral decubitus position using a Phillips iE33 Echocardiography machine with a $2.5 \mathrm{MHz}$ transducer. Images were acquired from apical four-chamber, two-chamber, and three-chamber views using two-dimensional (2D), M-mode, color Doppler, continuous-wave Doppler, and pulse-wave Doppler according to recommendations in the American Society of Echocardiography (ASE) guidelines ${ }^{[7,12]}$ The left ventricle (LV) and left atrium (LA) dimensions were evaluated in M-mode during LV diastole from 2D parasternal images. LA size and volume were also evaluated from apical four-chamber and two-chamber views at ventricular end-systole (modified Simpson's method). LA volume indices (LAVIs) were calculated by dividing the LA volumes by the body surface area. ${ }^{[7]}$ The sample volume of pulsed-wave Doppler was taken at the mitral leaflets' tip level from the apical four-chamber view for mitral inflow velocity assessment. The peak mitral inflow in early diastole ( $\mathrm{E}$ wave), the late diastolic atrial filling velocity (A wave), their ratio (E/A ratio), mitral deceleration time (DecT), and aortic ejection time (ET) parameters were measured. The sample volume with measurements of myocardial systolic ( $\left.\mathrm{S}^{\prime}\right)$, early $\left(\mathrm{E}^{\prime}\right)$, and late $\left(\mathrm{A}^{\prime}\right)$ diastolic velocities were applied at the septal and lateral sides of the mitral annulus using pulsed-wave tissue Doppler imaging (TDI) to assess myocardial velocities. All the TDI measures were obtained by averaging values at the septal and lateral mitral annulus. LV isovolumic contraction time (IVCT) and isovolumic relaxation time (IVRT) were acquired from the pulse wave Doppler traces. The Myocardial Performance Index (MPI) was calculated from the formula (IVCT x IVRT)/ET.

\section{Speckle-tracking echocardiography}

A specific software package, QLAB V6.0 (Advanced Quantification Software version 6; Phillips), was used to perform the STE analysis from the apical and parasternal short-axis offline images at a frame rate of 70-90 frames/s. Three consecutive cardiac cycles were apical four-chamber, two-chamber, three-chamber views, and parasternal short-axis basal, mid, and apical views were recorded. They averaged ${ }^{[13]}$ in Digital Imaging and Communications in Medicine format for offline STE analysis by an experienced cardiologist. Images were acquired at end-expiration during breath hold.

Records were examined in the region of interest (ROI) tab; then, the views were drawn to include the entire myocardium. The 
ROI thickness was the same in all the patients independently from LV hypertrophy.

The first step in this process was to mark the mitral annulus endocardial borders and apex on the apical two-, three-, and four-chamber images. Second, automatic tracing was performed [Figure 1]. Endocardial borders were reviewed during end-systolic frames, and images were settled in motion for tracking confirmation. Areas that cannot be tracked were excluded. Third, peak systolic strain was measured and averaged for determining GLS. Before conducting radial strain analysis, the anatomical structures were marked manually as anterior, inferior, and interventricular parts on the short-axis basal, mid, and apical images and endocardial borders were determined by software. Then, borders were again reviewed during end-systolic frames, and images were settled in motion for tracking confirmation. Peak systolic GCS and GRS values were calculated by averaging from analyzed ventricular segments. The software could track $95 \%$ of segments longitudinal for GLS and 93\% for GRS and GCS, respectively.

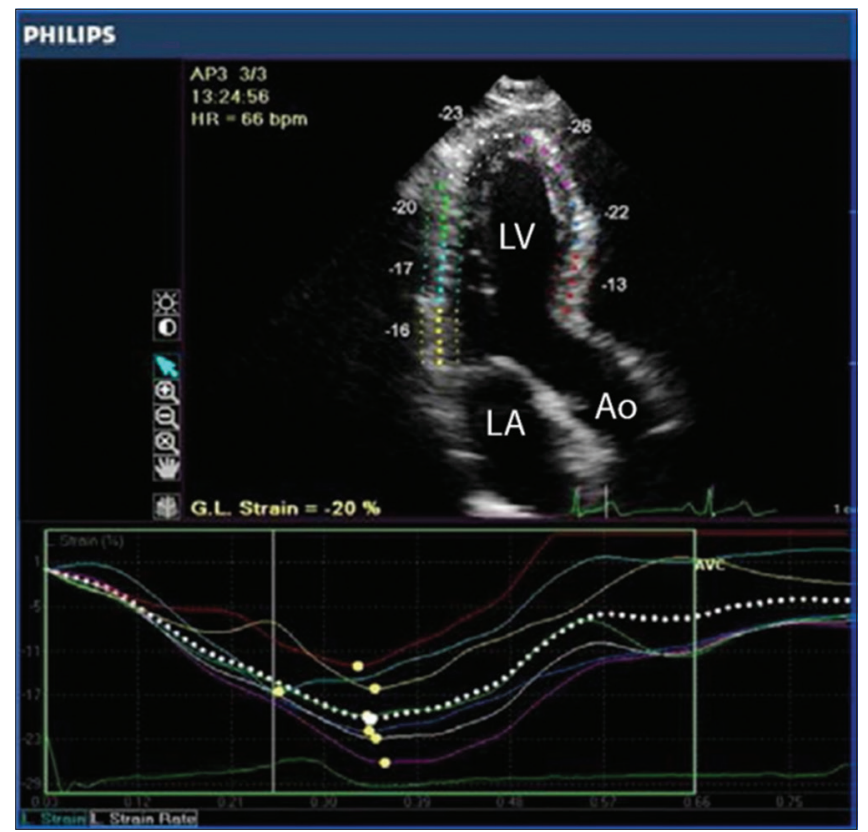

Figure 1: Determination of endocardial borders in speckle-tracking evaluation in a mild obstructive sleep apnea patient
Finally, a bullseye image showing left ventricle whole-strain parameters was obtained [Figure $2 \mathrm{a}$ and $\mathrm{b}$ ].

\section{Statistical analysis}

Data management and analysis were performed using SPSS version 15.0 (SPSS Inc., Chicago, IL, USA). The fitting of variables to normal distribution was carried out with analytical methods (Kolmogorov-Smirnov tests). The Student's $t$-test was used to compare parametric variables, and the Chi-square test was used to compare nonparametric variables. Descriptive analyses were presented using means and standard deviations for normally distributed variables. Since 2D echocardiographic data, diastolic parameters, and LV strain values were all determined to show normal distribution, these parameters were compared using a one-way ANOVA test between the OSA groups. The homogeneity of the variances was calculated using the Levene test.

Descriptive analyses were performed using the median and interquartile range for nonnormally distributed variables. Systolic blood pressure, posterior wall (PW) thickness, mitral inflow E/A ratio, and tissue Doppler mitral lateral annulus IVRT were determined to have normal distribution between these parameters in the OSA groups. The analysis was performed using the Kruskal-Wallis test and evaluated using the Bonferroni correction. $P<0.05$ was considered to show statistically significant results.

Correlation with the AHI was examined using the Pearson test. Those results with significant correlations were taken into the first univariable linear regression analysis (unadjusted), and variables found $P<0.05$ were considered in an ordered multivariate logistic regression model (adjusted).

\section{RESULTS}

Table 1 summarizes the demographic and clinical characteristics of the control and OSA groups. The two groups were comparable for sex $(P=0.450)$, age $(P=0.560)$, smoking $(P=0.970)$, and heart rate $(P=0.057)$. However, diabetes $(P<0.001)$ and hypertension $(P<0.001)$ diagnoses, body mass index $(P<0.001)$, and systolic $(P<0.001)$ and diastolic $(P=0.002)$ blood pressure were higher in the OSA patients than in the healthy controls.

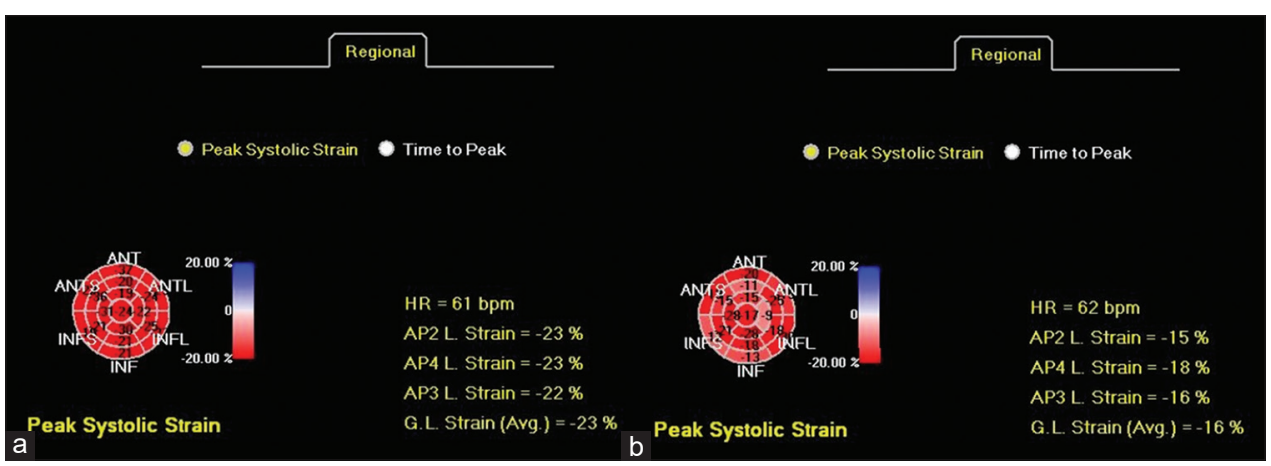

Figure 2: (a) Global longitudinal strain and bullseye image of a participant in control group. (b) Global longitudinal strain and bullseye image of severe obstructive sleep apnea patient 
Table 2 shows the standard echocardiographic characteristics of the subgroups. LV EF with Simpson's method was $63.12 \%$ $\pm 2.78 \%$ in the control group and $61.81 \% \pm 3.45 \%$ in the OSA group $(P<0.001)$. LV septal wall thickness $(P=0.007)$, LV

\begin{tabular}{lccc}
\multicolumn{4}{c}{ Table 1: Demographic, clinical variables of the all groups } \\
\hline & Control & OSA $(\boldsymbol{n}=\mathbf{7 9})$, & $\boldsymbol{P}$ \\
& $(\boldsymbol{n = 2 1 ) , \boldsymbol { n } ( \% )}$ & $\boldsymbol{n}(\%)$ & \\
\hline Age (years) & $49.2 \pm 8.6$ & $50.5 \pm 9.1$ & 0.560 \\
Sex (male/female) & $15 / 6$ & $48 / 31$ & 0.450 \\
Smoking & $7(33)$ & $23(29)$ & 0.970 \\
DM & 0 & $13(16)$ & $<0.001$ \\
HT & 0 & $31(39)$ & $<0.001$ \\
Hypothyroidism & 0 & $3(3.7)$ & 0.360 \\
BMI (kg/m $\left.{ }^{2}\right)$ & $25.5 \pm 3.4$ & $29.9 \pm 4.6$ & $<0.001$ \\
HR (beat/min) & $76.6 \pm 9.5$ & $81.9 \pm 11.9$ & 0.057 \\
SBP (mmHg) & $111.8 \pm 8.9$ & $124.5 \pm 11.5$ & $<0.001$ \\
DBP (mmHg) & $69.4 \pm 7.0$ & $75.9 \pm 8.9$ & 0.002 \\
AHI (IQR) & $0(0-3)$ & $35.6(8-22)$ & $<0.001$ \\
\hline
\end{tabular}

Data are expressed as, $n(\%)$ or mean \pm SD. OSA: Obstructive sleep apnea, DM: Diabetes mellitus, HT: Hypertension, BMI: Body mass index, HR: Heart rate, SBP: Systolic blood pressure, DBP: Diastolic blood pressure, AHI: Apnea-Hypopnea Index, IQR: Interquartile range, SD: Standard deviation internal dimensions $(P=0.023)$, and LV mass index $(P<0.001)$ were higher in the OSA subgroups. DecT $(P<80.001)$ and IVRT $(P<0.001)$ were also longer in OSA. While $\mathrm{E} / \mathrm{A}(P<0.001)$ and $\mathrm{E} / \mathrm{E}^{\prime}$ ratios $(P<0.001)$, which are parameters of diastolic function, were impaired in the OSA group. The MPI increased with OSA $(P<0.001)$. If we look at the estimated systolic pulmonary artery pressure (sPAP) of the two groups, it was measured as $23.09 \pm 3.80 \mathrm{mmHg}$ in the control group and $32.89 \pm 6.46 \mathrm{mmHg}$ in the OSA group $(P<0.001)$.

The next section of the study showed LV GLS (-\%13.32 \pm 3.19 vs. $-\% 18.60 \pm 4.60, P<0.001)$, GCS $(-\% 18.3 \pm 3.4$ vs. $-\% 22.27 \pm 2.40, P<0.001)$, and GRS $(37.9 \% \pm 8.1 \%$ vs. $42.47 \% \pm 5.00 \%, P=0.005)$ variables were decreased in OSA patients compared to the control group, as presented in Table 3. Figure 3 shows a clear trend of decreasing GLS and GCS as we moved from the control to the severe OSA patients, while GRS increased in mild and moderate OSA patients compared to the healthy group. Interestingly, GRS decreased in the severe OSA subgroup.

There was a significant positive correlation between AHI and GCS $(r=-0.53, P<0.001)$, GRS $(r=-0.68, P<0.001)$,

Table 2: Two-dimensional, M-mode. and Doppler echocardiographic measurements of the study population

\begin{tabular}{|c|c|c|c|c|c|}
\hline & Control $(n=21)$ & Mild OSA $(n=20)$ & Moderate OSA $(n=21)$ & Severe OSA $(n=38)$ & $P^{*}$ \\
\hline LA Vol (mL) & $35.72 \pm 3.16$ & $37.4 \pm 4.52$ & $37.91 \pm 3.15$ & $42.67 \pm 3.64$ & $<0.001$ \\
\hline LAVI $\left(\mathrm{mL} / \mathrm{m}^{2}\right)$ & $19.63 \pm 4.61$ & $21.15 \pm 5.35$ & $25.54 \pm 6.52$ & $31.51 \pm 6.11$ & $<0.001$ \\
\hline LV EF (\%) & $63.12 \pm 2.78$ & $62.60 \pm 3.60$ & $61.88 \pm 3.20$ & $60.95 \pm 3.55$ & 0.008 \\
\hline LV EDV (mL) & $88.37 \pm 5.24$ & $88.95 \pm 3.31$ & $89.12 \pm 3.59$ & $90.22 \pm 4.32$ & 0.101 \\
\hline LV ESV (mL) & $32.18 \pm 2.15$ & $32.83 \pm 3.24$ & $32.76 \pm 3.21$ & $33.92 \pm 3.51$ & 0.023 \\
\hline IVS (mm) & $10.32 \pm 1.14$ & $10.29 \pm 1.43$ & $11.44 \pm 1.31$ & $11.91 \pm 1.59$ & $<0.001$ \\
\hline $\mathrm{PW}(\mathrm{mm})$ & $9.56 \pm 1.34$ & $9.58 \pm 0.98$ & $10.08 \pm 1.25$ & $10.75 \pm 1.45$ & 0.001 \\
\hline $\operatorname{LVDD}(\mathrm{mm})$ & $48.32 \pm 4.43$ & $48.85 \pm 5.12$ & $49.48 \pm 4.85$ & $49.85 \pm 3.81$ & 0.527 \\
\hline $\operatorname{LVsD}(\mathrm{mm})$ & $30.61 \pm 1.39$ & $31.39 \pm 2.81$ & $32.18 \pm 2.82$ & $32.67 \pm 2.69$ & 0.023 \\
\hline LVMI & $88.51 \pm 14.42$ & $91.48 \pm 24.30$ & $105.51 \pm 26.92$ & $110.10 \pm 25.12$ & 0.002 \\
\hline $\mathrm{E}(\mathrm{cm} / \mathrm{sn})$ & $7.62 \pm 1.3$ & $7.48 \pm 1.09$ & $7.39 \pm 1.53$ & $6.99 \pm 1.33$ & 0.304 \\
\hline $\mathrm{A}(\mathrm{cm} / \mathrm{sn})$ & $6.46 \pm 1.89$ & $7.52 \pm 1.21$ & $8.14 \pm 1.31$ & $8.78 \pm 1.56$ & $<0.001$ \\
\hline $\operatorname{DecT}(\mathrm{s})$ & $169.23 \pm 13.20$ & $177.45 \pm 31.53$ & $189.45 \pm 33.12$ & $219.61 \pm 25.31$ & $<0.001$ \\
\hline $\mathrm{ET}(\mathrm{s})$ & $281.71 \pm 21.34$ & $271.65 \pm 35.87$ & $261.85 \pm 44.85$ & $249.68 \pm 23.42^{\dagger}$ & 0.002 \\
\hline E/A ratio & $1.33 \pm 0.70$ & $1.02 \pm 0.22^{\dagger}$ & $0.92 \pm 0.21^{\dagger}$ & $0.82 \pm 0.26^{\dagger}$ & $<0.001$ \\
\hline $\mathrm{E} / \mathrm{E}^{\prime}$ ratio & $6.53 \pm 1.63$ & $6.88 \pm 1.98$ & $7.92 \pm 2.43$ & $10.58 \pm 2.10^{\dagger, * * * *}$ & $<0.001$ \\
\hline IVRT (s) & $86.42 \pm 14.81$ & $110.05 \pm 22.62$ & $114.80 \pm 21.06^{\dagger}$ & $119.18 \pm 20.47^{\dagger}$ & $<0.001$ \\
\hline $\mathrm{S}^{\prime}$ wave $(\mathrm{cm} / \mathrm{sn})$ & $11.17 \pm 1.87$ & $10.02 \pm 2.21$ & $10.02 \pm 2.00$ & $9.30 \pm 1.90^{\dagger}$ & 0.010 \\
\hline $\mathrm{E}^{\prime}$ wave $(\mathrm{cm} / \mathrm{sn})$ & $12.11 \pm 2.57$ & $11.72 \pm 3.56$ & $10.06 \pm 3.15$ & $9.41 \pm 2.35^{\dagger, *}$ & 0.001 \\
\hline $\mathrm{A}^{\prime}$ wave $(\mathrm{cm} / \mathrm{sn})$ & $11.41 \pm 2.38$ & $12.19 \pm 1.90$ & $12.84 \pm 2.63$ & $13.04 \pm 2.04$ & 0.010 \\
\hline TDI IVCT & $48.00 \pm 7.25$ & $46.91 \pm 9.64$ & $52.15 \pm 12.05$ & $57.68 \pm 9.34$ & $<0.001$ \\
\hline TDI IVRT & $90.33 \pm 9.89$ & $98.00 \pm 18.55$ & $109.55 \pm 19.65$ & $112.28 \pm 19.37$ & $<0.001$ \\
\hline TDI ET & $281.52 \pm 13.95$ & $284.65 \pm 27.53$ & $270.95 \pm 23.10$ & $269.31 \pm 27.69$ & 0.093 \\
\hline LV MPI & $0.46 \pm 0.11$ & $0.60 \pm 0.15$ & $0.66 \pm 0.14$ & $0.71 \pm 0.11$ & $<0.001$ \\
\hline sPAP $(\mathrm{mmHg})$ & $23.09 \pm 3.80$ & $30.80 \pm 7.2$ & $32.20 \pm 4.34$ & $34.36 \pm 6.76$ & $<0.001$ \\
\hline
\end{tabular}

${ }^{\dagger}$ Compared with control group, $P<0.05$, ${ }^{*}$ Compared with mild OSA group, $P<0.05,{ }^{*}$ Compared with moderate OSA group, $P<0.05$, ${ }^{*}$ Tested with one-way ANOVA and Kruskal-Wallis, post hoc comparisons made with Tukey's and Mann-Whitney U-test and evaluated with Bonferroni correction, ${ }^{\dagger}$ Compared to the control group $P<0.05$. Data are expressed as, $n(\%)$ or mean \pm SD. OSA: Obstructive sleep apnea, LAVI: Left atrial volume index, IVS: Interventricular septum, PW: Posterior wall, LV: Left ventricular, LVDD: LV diastolic dimension, LVsD: LV systolic dimension, LV EF: LV ejection fraction, with Simpson's method, LVMI: LV mass index, LV ESV: LV end-systolic volume, LV EDV: LV end-diastolic volume, DecT: Deceleration time, TDI: Tissue Doppler imaging, ET: Ejection time, IVRT: Isovolumic relaxation time, IVCT: Isovolumic contraction time, LV MPI: LV myocardial performance index, sPAP: Systolic pulmonary pressure, SD: Standard deviation 
GLS $(r=-0.62, P<0.001)$, interventricular septum thickness (IVS) $(r=0.40, P<0.001), \mathrm{PW}(r=0.35, P<0.001)$, sPAP $(r=0.46, P<0.001)$, LAVI $(r=0.28, P=0.013)$, $\mathrm{E} / \mathrm{E} '(r=0.51, P<0.001)$, LV MPI $(r:=0.23, P=0.041)$, and DecT $(r=0.48, P<0.001)$ variables [Table 4 ].

Linear regression analysis was performed to determine the parameters that correlate independently with the AHI. GRS ( $\beta$ : $-1.47 ; 95 \%$ confidence interval $[\mathrm{CI}]:-1.97-0.98, P=0.001)$, GCS $(\beta:-1.34 ; 95 \%$ CI: $-2.04-1.10, P=0.001)$, GLS $(\beta$ : -1.54 ; 95\% CI: $-1.93-1.04, P<0.001), \operatorname{sPAP}(\beta: 0.99,95 \% \mathrm{CI}$ : $0.49-1.53, P=0.001)$, and $\operatorname{DecT}(\beta: 0.13 ; 95 \%$ CI: $0.20-0.24$, $P=0.03$ ) were found to be independently associated with AHI [Table 4].

\section{Discussion}

OSA is frequently associated with many cardiovascular diseases and severe clinical outcomes, including heart failure. The current study's main goal was to evaluate LV functions using STE-based analyses in OSA patients before developing the overt clinical disease. We found that GLS, GCS, and GRS values decreased in OSA patients compared to a healthy control group, while LV functions were still intact in 2D echocardiographic imaging. One interesting finding was that GRS increased in mild and moderate OSA patients while it decreased significantly in the severe OSA group. However, GLS and GCS were decreasing from the control to severe OSA groups. The second goal of this research was to look for LV diastolic parameters and LV hypertrophic changes in OSA patients compared to the control group. Diastolic functions were found impaired with the OSA progression; in addition, the LV was more hypertrophic in OSA patients. Another objective of the study was to determine independent factors for AHI. We found that GLS, GCS, GRS, sPAP, and DecT have an independent relationship with AHI.

Prior studies have noted the significance of heart failure development in OSA patients compared with the general population. ${ }^{[14-16]}$ However, the traditional systolic function parameter LV EF was normal in most OSA patients despite heart failure signs. ${ }^{[17,18]}$ In addition, it is difficult to detect cardiac changes with conventional 2D echocardiography. GLS is a recent marker for subclinical LV pump dysfunction, and we found that GLS was decreasing from the healthy control group toward severe OSA patients. This result seems to be consistent with other researches. ${ }^{[18-20]}$ A possible explanation for the decrease in LV longitudinal strain may be the susceptibility of subendocardial fibers to hypoxia in OSA patients.

Furthermore, a higher prevalence of diabetes and hypertension in the OSA groups could affect LV longitudinal function GLS even with preserved diastolic function. Myocardial longitudinal shortening and myocyte apoptosis cause a decrease in longitudinal and circumferential strain values. While GLS and GCS decrease in the mild OSA group, GRS increases. The reason for this finding is not apparent, but it may be associated with adaptive mechanisms such as

\begin{tabular}{|c|c|c|c|}
\hline & Control $(n=21)$ & OSA $(n=79)$ & $P$ \\
\hline GLS (\%) & $-18.60 \pm 4.60$ & $13.32 \pm 3.19$ & $<0.001$ \\
\hline GCS (\%) & $-22.27 \pm 2.40$ & $18.33 \pm 3.40$ & $<0.001$ \\
\hline GRS (\%) & $42.47 \pm 5.00$ & $37.91 \pm 8.11$ & 0.005 \\
\hline
\end{tabular}

Data are expressed as, $n(\%)$ or mean \pm SD. OSA: Obstructive sleep apnea, GLS: Global longitudinal strain, GCS: Global circumferential strain, GRS: Global radial strain, SD: Standard deviation

Table 4: Linear regression analysis of Apnea-Hypopnea Index with clinical and echocardiographic measurements

\begin{tabular}{|c|c|c|c|c|c|c|}
\hline & \multirow[t]{2}{*}{$r$} & \multirow[t]{2}{*}{$P$} & \multirow[t]{2}{*}{$\beta$} & \multicolumn{2}{|c|}{$\mathrm{Cl}$} & \multirow[t]{2}{*}{$P$} \\
\hline & & & & $\begin{array}{l}\text { Lower } \\
\text { bound }\end{array}$ & $\begin{array}{l}\text { Upper } \\
\text { bound }\end{array}$ & \\
\hline Age & 0.7 & 0.015 & - & - & - & - \\
\hline SBP & 0.32 & 0.004 & - & - & - & - \\
\hline DBP & 0.22 & 0.043 & - & - & - & - \\
\hline GCS & -0.53 & $<0.001$ & -1.34 & -2.04 & -1.10 & 0.001 \\
\hline GRS & -0.68 & $<0.001$ & -1.47 & -1.97 & -0.98 & 0.001 \\
\hline GLS & -0.62 & $<0.001$ & -1.54 & -1.93 & -1.04 & 0.001 \\
\hline IVS & 0.40 & $<0.001$ & - & - & - & - \\
\hline PW & 0.35 & $<0.001$ & - & - & - & - \\
\hline sPAP & 0.46 & $<0.001$ & 0.99 & 0.49 & 1.53 & 0.001 \\
\hline DecT & 0.48 & $<0.001$ & 0.13 & 0.20 & 0.24 & 0.031 \\
\hline IVCT & 0.23 & 0.035 & - & - & - & - \\
\hline LV MPI & 0.23 & 0.041 & - & - & - & - \\
\hline LAVI & 0.28 & 0.013 & - & - & - & - \\
\hline E/E' & 0.51 & $<0.001$ & - & - & - & - \\
\hline
\end{tabular}

SBP: Systolic blood pressure, DBP: Diastolic blood pressure, GLS: Global longitudinal strain, GCS: Global circumferential strain, GRS: Global radial strain, IVS: Interventricular septum, PW: Posterior wall, sPAP: Systolic pulmonary artery pressure, DecT: Deceleration time, IVCT: Isovolumic contraction time, LV: Left ventricular, LV MPI: LV myocardial performance index, LAVI: Left atrial volume index, CI: Confidence interval

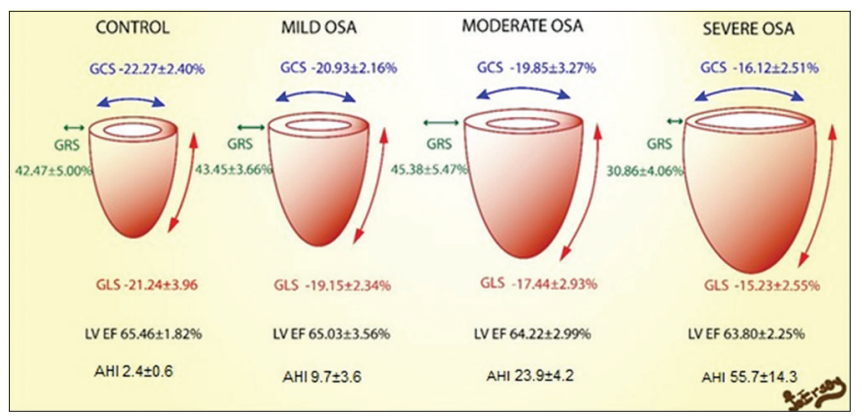

Figure 3: An illustration showing global strain (GLS, GRS, and GCS) patterns changing normal to severe OSA. Data are expressed as number (\%) or mean \pm SD. Abbreviations: OSA: Obstructive sleep apnea, GLS: Global longitudinal strain, GCS: Global circumferential strain, GRS: Global radial strain, AHI: Apnea-Hypopnea Index, SD: Standard deviation

radial thickening to preserve LV EF. In the later stages of OSA, all strain parameters decreased significantly compared to the control group. This situation's main reason may be increased cardiac afterload, and LV adaptive mechanisms are not responding to this condition and causing LV subclinical 
dysfunction. There are investigations in which TDI has been used to represent an early stage of myocardial abnormality despite preserved global LV function. ${ }^{[17,21,22]}$ The TDI is an angle-dependent technique, and it has significant test variabilities, causing important limitations. STE does not need angle interrogation ${ }^{[23]}$ All global strain value reduction broadly supports the results of other studies in this area linking OSA with subclinical dysfunction. ${ }^{[10,18,19]}$

When we look at the second aim of this study, our results showed relevant connections between OSA and LV hypertrophy, LV mass index diastolic dysfunctions, and enlargement of the left ventricle and atrium, consistent with other researches. ${ }^{[24-26]} \mathrm{In}$ our results, as the OSA severity increased from the healthy control group, LV hypertrophy, LA diameter, LV diameter, LV mass index, and LV MPI also increased. We found that as for diastolic parameters, like E, E/A ratio decreased, A, E/E' ratio and IVRT, DecT, and ET elevated in OSA progression. These findings can be explained with some hypotheses: compensatory changes occur against an increase in LV afterload; nocturnal hypoxia and sympathetic hyperactivity can increase blood pressure; and respiratory effort during apnea can cause severe negative intrathoracic pressure, resulting in raised transmural pressure. These data must be interpreted with caution because our study participants tended to be obese, hypertensive, and diabetic. These factors are well-known causes of LV hypertrophy and diastolic dysfunction.

Another significant aspect of our study underlines that the AHI, which reflects OSA severity, correlates with GRS, GCS, E/E' ratio, DecT, IVS, PW, and SPAP. Patients with a higher AHI had more impaired horizontal strain patterns and diastolic reserve. These relationships may partly be explained by nocturnal apnea and hypoxia episodes increasing LV afterload during sleep. This mechanism could be the first step of cardiac remodeling and result in impaired global strain patterns in OSA patients.

Our study excluded situations that could increase pulmonary pressure, such as chronic pulmonary disease and asthma. In our finding, sPAP was higher in OSA patients than healthy controls, in agreement with previous studies. ${ }^{[27,28]}$ In a physio-pathogenetic view, repetitive hypoxia can cause pulmonary vasoconstriction, while endothelial remodeling increases endothelin-1 release, NO release decreases, and OSA-related pulmonary pressure increases. ${ }^{[29]}$

Our study has some limitations. The main drawback was a single-center experience, and one cardiologist performed the echocardiography. This situation cannot exclude selection or procedure bias. Our control group had no hypertension or diabetes, and the OSA patients were overweight individuals. Hypertension, diabetes, and obesity could affect diastolic and LV dimension results. Furthermore, this work is also limited by not considering medications like renin-angiotensin antagonists that could affect early remodeling. In addition, the severe OSA group included the highest number of patients. Our study lacks hemodynamic data. An assessment by right heart catheterization might have provided more accurate information about sPAP. This study's final limitation relates to not considering markers such as B-type natriuretic peptide and $\mathrm{N}$-terminal pro-brain natriuretic peptide diagnosing heart failure.

Strain parameters are a proven method that has entered guidelines and consensus documents, as in the ASE and the European Association of Cardiovascular Imaging. ${ }^{[30]}$ Merely one of its main limitations, which is the time-consuming offline analysis, might make it difficult to apply STE to every clinical setting, particularly to the outpatient clinic. Furthermore, there is no agreement on STE strain parameters for OSA presence and severity. Future follow-up studies seeking to use STE should attempt to determine predictive LV strain value on OSA patients' outcomes.

\section{Conclusions}

Cardiac mechanic evaluation with STE will be a regular part of the echocardiographic imaging. Furthermore, impaired cardiac mechanics project the first step in the complex cascade of cardiac remodeling in OSA patients. Our study results indicate that LV strains and diastolic functions were impaired in OSA patients while LV EF was in the normal range. STE technique can be a practical method for manifesting LV functional impairment at the newly diagnosed stages of OSA.

\section{Ethical statement}

The study was conducted under the Declaration of Helsinki. The Ethical Committee approved the study for Clinical Studies of Suleyman Demirel University (Registration Number: 181). All participants gave written informed consent.

\section{Acknowledgments}

The present study work has been presented as an abstract at the International Young Academy of Cardiology Congress 2019.

\section{Financial support and sponsorship}

Nil.

\section{Conflicts of interest}

There are no conflicts of interest.

\section{References}

1. Peppard PE, Young T, Barnet JH, Palta M, Hagen EW, Hla KM. Increased prevalence of sleep-disordered breathing in adults. Am J Epidemiol 2013;177:1006-14.

2. Benjafield AV, Ayas NT, Eastwood PR, Heinzer R, Ip MS, Morrell MJ, et al. Estimation of the global prevalence and burden of obstructive sleep apnoea: A literature-based analysis. Lancet Respir Med 2019;7:687-98.

3. Borrelli C, Gentile F, Sciarrone P, Mirizzi G, Vergaro G, Ghionzoli N, et al. Central and obstructive apneas in heart failure with reduced, mid-range and preserved ejection fraction. Front Cardiovasc Med 2019;6:125.

4. Herrscher TE, Akre H, Øverland B, Sandvik L, Westheim AS. High prevalence of sleep apnea in heart failure outpatients: Even in patients with preserved systolic function. J Card Fail 2011;17:420-5.

5. Sascău R, Zota IM, Stătescu C, Boișteanu D, Roca M, Maștaleru A, et al. Review of echocardiographic findings in patients with obstructive sleep apnea. Can Respir J 2018;2018:1206217. 1-9.

6. Cameli M, Mandoli GE, Sciaccaluga C, Mondillo S. More than 10 years 
of speckle tracking echocardiography: Still a novel technique or a definite tool for clinical practice? Echocardiography 2019;36:958-70.

7. Lang RM, Bierig M, Devereux RB, Flachskampf FA, Foster E, Pellikka PA, et al. Recommendations for chamber quantification: A report from the American Society of Echocardiography's Guidelines and Standards Committee and the Chamber Quantification Writing Group, developed in conjunction with the European Association of Echocardiography, a branch of the European Society of Cardiology. J Am Soc Echocardiogr 2005;18:1440-63.

8. Opdahl A, Helle-Valle T, Skulstad H, Smiseth OA. Strain, strain rate, torsion, and twist: Echocardiographic evaluation. Curr Cardiol Rep 2015; $17: 568$.

9. Sareen N, Ananthasubramaniam K. Strain imaging: From physiology to practical applications in daily practice. Cardiol Rev 2016;24:56-69.

10. Cuspidi C, Tadic M. Obstructive sleep apnea and left ventricular strain: Useful tool or fancy gadget? J Clin Hypertens (Greenwich) 2020;22:120-2.

11. Berry RB, Budhiraja R, Gottlieb DJ, et al. Rules for scoring respiratory events in sleep: update of the 2007 AASM Manual for the Scoring of Sleep and Associated Events. Deliberations of the Sleep Apnea Definitions Task Force of the American Academy of Sleep Medicine. J Clin Sleep Med 2012;8:597-619.

12. Nagueh SF, Smiseth OA, Appleton CP, Byrd BF, Dokainish H, Edvardsen $\mathrm{T}$, et al. Recommendations for the evaluation of left ventricular diastolic function by echocardiography: An update from the American Society of Echocardiography and the European Association of Cardiovascular Imaging. J Am Soc Echocardiogr 2016;29:277-314.

13. Voigt JU, Pedrizzetti G, Lysyansky P, Marwick TH, Houle H, Baumann R, et al. Definitions for a common standard for 2D speckle tracking echocardiography: Consensus document of the EACVI/ASE/ Industry Task Force to standardize deformation imaging. Eur Heart $\mathrm{J}$ Cardiovasc Imaging 2015;16:1-1.

14. Leung RS, Bradley TD. State of the art sleep apnea and cardiovascular disease. Crit Care Med 2001;164:2147-65.

15. Corrà U, Pistono $\mathrm{M}$, Mezzani $\mathrm{A}$, Braghiroli $\mathrm{A}$, Giordano $\mathrm{A}$, Lanfranchi P, et al. Sleep and exertional periodic breathing in chronic heart failure: Prognostic importance and interdependence. Circulation 2006;113:44-50

16. Gottlieb DJ, Yenokyan G, Newman AB, O'Connor GT, Punjabi NM, Quan SF, et al. Prospective study of obstructive sleep apnea and incident coronary heart disease and heart failure: The sleep heart health study. Circulation 2010;122:352-60.

17. Wachter R, Lüthje L, Klemmstein D, Lüers C, Stahrenberg R, Edelmann $\mathrm{F}$, et al. Impact of obstructive sleep apnoea on diastolic function. Eur Respir J 2013;41:376-83.

18. Zhou NW, Shu XH, Liu YL, Shen H, Li WJ, Gong X, et al. A novel method for sensitive determination of subclinical left-ventricular systolic dysfunction in subjects with obstructive sleep apnea. Respir Care 2016;61:366-75.

19. Altekin RE, Yanikoglu A, Baktir AO, Karakas MS, Ozel D, Cilli A, et al. Assessment of subclinical left ventricular dysfunction in obstructive sleep apnea patients with speckle tracking echocardiography. Int J Cardiovasc Imaging 2012;28:1917-30.

20. Varghese MJ, Sharma G, Shukla G, Seth S, Mishra S, Gupta A, et al. Longitudinal ventricular systolic dysfunction in patients with very severe obstructive sleep apnea: A case control study using speckle tracking imaging. Indian Heart J 2017;69:305-10.

21. Kim SH, Cho GY, Shin C, Lim HE, Kim YH, Song WH, et al. Impact of obstructive sleep apnea on left ventricular diastolic function. Am J Cardiol 2008;101:1663-8.

22. Haruki N, Takeuchi M, Nakai H, Kanazawa Y, Tsubota N, Shintome R, et al. Overnight sleeping induced daily repetitive left ventricular systolic and diastolic dysfunction in obstructive sleep apnoea: Quantitative assessment using tissue Doppler imaging. Eur J Echocardiogr 2009;10:769-75.

23. Dandel M, Hetzer R. Echocardiographic strain and strain rate imaging - Clinical applications. Int J Cardiol 2009;132:11-24.

24. Kim SM, Cho KI, Kwon JH, Lee HG, Kim TI. Impact of obstructive sleep apnea on left atrial functional and structural remodeling beyond obesity. J Cardiol 2012;60:475-83.

25. Otto ME, Belohlavek M, Romero-Corral A, Gami AS, Gilman G, Svatikova A, et al. Comparison of cardiac structural and functional changes in obese otherwise healthy adults with versus without obstructive sleep apnea. Am J Cardiol 2007;99:1298-302.

26. Altekin RE, Yanıkoğlu A, Karakaș MS, Ozel D, Yıldırım AB, Kabukçu M. Evaluation of subclinical left ventricular systolic dysfunction in patients with obstructive sleep apnea by automated function imaging method; an observational study. Anadolu Kardiyol Derg 2012;12:320-30.

27. Chaouat A, Weitzenblum E, Krieger J, Oswald M, Kessler R. Pulmonary hemodynamics in the obstructive sleep apnea syndrome. Results in 220 consecutive patients. Chest 1996;109:380-6.

28. Yamakawa H, Shiomi T, Sasanabe R, Hasegawa R, Ootake K, Banno K, et al. Pulmonary hypertension in patients with severe obstructive sleep apnea. Psychiatry Clin Neurosci 2002;56:311-2.

29. Phillips BG, Narkiewicz K, Pesek CA, Haynes WG, Dyken ME, Somers VK. Effects of obstructive sleep apnea on endothelin-1 and blood pressure. J Hypertens 1999;17:61-6.

30. Lang RM, Badano LP, Mor-Avi V, Afilalo J, Armstrong A, Ernande L, et al. Recommendations for cardiac chamber quantification by echocardiography in adults: An update from the American Society of Echocardiography and the European Association of Cardiovascular Imaging. Eur Heart J Cardiovasc Imaging 2015;16:233-70. 